Journal of Universal Mathematics

Vol.4 No.2 PP.118-127 (2021)

ISSN-2618-5660

DOI: $10.33773 /$ jum. 962057

\title{
BLOW UP AT INFINITY OF WEAK SOLUTIONS FOR A HIGHER-ORDER PARABOLIC EQUATION WITH LOGARITHMIC NONLINEARITY
}

\author{
TUĞRUL CÖMERT AND ERHAN PIŞKIN \\ 0000-0002-8176-332X and 0000-0001-6587-4479
}

\begin{abstract}
The main goal of this work is to study the inital boundary value problem for a higher-order parabolic equation with logarithmic source term

$$
u_{t}+(-\Delta)^{m} u=u \ln |u| \text {. }
$$

We obtain blow-up at $+\infty$ of weak solutions, by employing potential well technique. This improves and extends some previous studies.
\end{abstract}

\section{INTRODUCTION}

In this paper, we cosider the following higher-order parabolic problem with logarithmic nonlinearity

$$
\begin{cases}u_{t}+A u=u \ln |u|, & x \in \Omega, \quad t>0, \\ D^{\gamma} u(x, t)=0, \quad|\gamma| \leq m-1, & x \in \partial \Omega, \quad t>0, \\ u(x, 0)=u_{0}(x), & x \in \Omega,\end{cases}
$$

where $A=(-\Delta)^{m}, m \geq 1$ a positive integer, $\Omega$ is a bound domain in $\mathbb{R}^{n}$ with smooth boundary $\partial \Omega, \gamma=\left(\gamma_{1}, \gamma_{2}, \ldots, \gamma_{n}\right)$ is multi-index, $\gamma_{i}(i=1,2, \ldots, n)$ are nonnegative integers, $|\gamma|=\gamma_{1}+\gamma_{2}+\ldots+\gamma_{n}, D^{\gamma}=\frac{\partial|\gamma|}{\partial x_{1}^{\gamma 1} \partial x_{2}^{\gamma 2} \ldots \partial x_{n}^{\gamma_{n}}}$ are multi-index derivative operator, $\Delta=\sum_{i=1}^{n} \frac{\partial^{2}}{\partial x_{i}^{2}}$ is the Laplace operator.

When $m=1$, the equation (1.1) becomes a heat equation as follows

$$
u_{t}-\Delta u=u \ln |u|
$$

In the equation (1.2), Chen et al. [2] obtained under some suitable conditions for the global existence, decay estimate and blow-up at $+\infty$ of weak solutions, via the logarithmic Sobolev inequality and potential well technique. Also, Han [5] obtained the blow-up at infinity of solutions, via the logarithmic Sobolev inequality. Additionally, Chen and Tian [3] obtained the global existence of solution, blow-up at $+\infty$ of solution, by adding strong damping term to the equation (1.2).

Date: Received: 2021-07-03; Accepted: 2021-07-27 .

2000 Mathematics Subject Classification. 35B40, 35G25, 35K35.

Key words and phrases. Higher-order parabolic equation, Blow up, Logarithmic nonlinearity. 
Peng and Zhou [10] studied the following semilinear heat equation with logarithmic nonlinearity

$$
u_{t}-\Delta u=u^{p-2} u \ln |u|,
$$

where $2<p$. They studied the existence of the unique global weak solutions and blow-up in the finite time of weak solutions, via potential well technique and energy technique.

Li and Liu [8] established a class of fourth-order parabolic equation with logarithmic source term as follows

$$
u_{t}+\Delta^{2} u=u^{p-2} u \ln |u|,
$$

where $2<p$. They studied the existence of global solutions, by using potential well technique. In addition, they also studied result of decay and finite time blow-up for weak solutions.

Nhan and Truong [9] studied the following nonlinear pseudo-parabolic equation

$$
u_{t}-\Delta u_{t}-\operatorname{div}\left(|\nabla u|^{p-2} \nabla u\right)=|u|^{p-2} u \log |u| .
$$

They obtained results as regard the existence or non-existence of global solutions. Also, He et al. [6] proved the decay and the finite time blow-up for weak solutions of the equation.

Resently many other authors investigated higher-order hyperbolic and parabolic type equation $[4,7,11,12,13,14,15]$. Ishige et al. [7] studied the Cauchy problem for nonlinear higher-order heat equation as follows

$$
u_{t}+(-\Delta)^{m} u=|u|^{p} .
$$

They obtained existence of solutions of the Cauchy problem by introducing a new majorizing kernel. In addition, they studied the local existence of solutions under the different conditions.

Xiao and $\mathrm{Li}[13]$ considered initial boundary value problem for nonlinear higherorder heat equations of

$$
u_{t}+(-\Delta)^{m} u_{t}+(-\Delta)^{m} u=f(u) .
$$

They established the existence of weak solution to the static problem, by using the potential well technique.

The remainder of our work is organized as follows. In Section2, some important Lemmas are given. In Section 3, the main result is proved.

\section{Preliminaries}

$$
\text { Let }\|u\|_{H^{m}(\Omega)}=\left(\sum_{|\gamma| \leq m}\left\|D^{\gamma} u\right\|_{L^{2}(\Omega)}^{2}\right)^{\frac{1}{2}} \operatorname{denote} H^{m}(\Omega) \text { norm, let } H_{0}^{m}(\Omega) \text { denote }
$$

the closure in $H^{m}(\Omega)$ of $C_{0}^{\infty}(\Omega)$. Let $\|\cdot\|_{r}$ and $\|\cdot\|$ denote the usual $L^{r}(\Omega)$ norm and $L^{2}(\Omega)$ norm.

For $u \in H_{0}^{m}(\Omega) \backslash\{0\}$, we define the energy functional

$$
J(u)=\frac{1}{2}\left\|A^{\frac{1}{2}} u\right\|^{2}-\frac{1}{2} \int_{\Omega}|u|^{2} \ln |u| d x+\frac{1}{4}\|u\|^{2},
$$

and Nehari functional

$$
I(u)=\left\|A^{\frac{1}{2}} u\right\|^{2}-\int_{\Omega}|u|^{2} \ln |u| d x .
$$


By (2.1) and (2.2), we obtain

$$
J(u)=\frac{1}{2} I(u)+\frac{1}{4}\|u\|^{2} .
$$

Further, let

$$
d=\inf _{u \in \mathcal{N}} J(u),
$$

denote the potential depth, where $\mathcal{N}$ is the Nehari manifold, which is defined by

$$
\mathcal{N}=\left\{u \in H_{0}^{m}(\Omega) \backslash\{0\}: I(u)=0\right\} .
$$

Lemma 2.1. [1]. Let $k$ be a number with $2 \leq k<+\infty, n \leq 2 m$ and $2 \leq k \leq \frac{2 n}{n-2 m}$, $n>2 m$. Then there is a constant $C$ depending

$$
\|u\|_{k} \leq C\left\|A^{\frac{1}{2}} u\right\|, \quad \forall u \in H_{0}^{m}(\Omega) .
$$

Lemma 2.2. $J(t)$ is a nonincreasing function for $t \geq 0$ and

$$
J^{\prime}(u)=-\int_{\Omega} u_{t}^{2} d x \leq 0 .
$$

Proof. Multiplying the equation (1.1) by $u_{t}$ and integrating on $\Omega$, we get

$$
\int_{\Omega} u_{t}^{2} d x+\int_{\Omega} A u u_{t} d x=\int_{\Omega} u u_{t} \ln |u| d x .
$$

By straightforward calculation, we obtain

$$
\int_{\Omega} u_{t}^{2} d x+\frac{1}{2} \frac{d}{d t}\left\|A^{\frac{1}{2}} u\right\|^{2}=\frac{1}{2} \frac{d}{d t} \int_{\Omega}|u|^{2} \ln |u| d x-\frac{1}{4} \frac{d}{d t}\|u\|^{2},
$$

which yields that

$$
\frac{1}{2} \frac{d}{d t}\left\|A^{\frac{1}{2}} u\right\|^{2}-\frac{1}{2} \frac{d}{d t} \int_{\Omega}|u|^{2} \ln |u| d x+\frac{1}{4} \frac{d}{d t}\|u\|_{2}^{2}=-\int_{\Omega} u_{t}^{2} d x .
$$

Thus, we get

$$
\frac{d}{d t}\left(\frac{1}{2}\left\|A^{\frac{1}{2}} u\right\|^{2}-\frac{1}{2} \int_{\Omega}|u|^{2} \ln |u| d x+\frac{1}{4}\|u\|_{2}^{2}\right)=-\int_{\Omega} u_{t}^{2} d x .
$$

By 2.1 and 2.6, we obtain

$$
\frac{d}{d t} J(u)=-\int_{\Omega} u_{t}^{2} d x .
$$

Moreover, Integrating (2.7) with respect to $t$ on $[0, t]$, we arrive at

$$
\int_{0}^{t}\left\|u_{s}(s)\right\|^{2} d s+J\left(u(t)=J\left(u_{0}\right)\right.
$$

Lemma 2.3. Let $u \in H_{0}^{m}(\Omega) \backslash\{0\}$ and $j(\lambda)=J(\lambda u)$. Then we get

(i) $\lim _{\lambda \rightarrow 0^{+}} j(\lambda)=0$ and $\lim _{\lambda \rightarrow+\infty} j(\lambda)=-\infty$,

(ii) there is a unique $\lambda^{*}>0$ such that $j^{\prime}\left(\lambda^{*}\right)=0$, 
(iii) $j(\lambda)$ is increasing on $\left(0, \lambda^{*}\right)$, decreasing on $\left(\lambda^{*},+\infty\right)$ and taking the maximum at $\lambda^{*}$,

(iv) $I(\lambda u)>0$ for $\lambda \in\left(0, \lambda^{*}\right), I(\lambda u)<0$ for $\lambda \in\left(\lambda^{*},+\infty\right)$ and $I\left(\lambda^{*} u\right)=0$.

Proof. By the definition of $j$, for $u \in H_{0}^{1}(\Omega) \backslash\{0\}$, we get

$$
j(\lambda)=\frac{\lambda^{2}}{2}\left\|A^{\frac{1}{2}} u\right\|^{2}-\frac{\lambda^{2}}{2} \int_{\Omega}|u|^{2} \ln |u| d x-\frac{\lambda^{2}}{2} \ln \lambda\|u\|_{2}^{2}+\frac{\lambda^{2}}{4}\|u\|^{2} .
$$

By (2.9), we have

$$
\begin{aligned}
\frac{d}{d \lambda} j(\lambda)= & \lambda\left\|A^{\frac{1}{2}} u\right\|^{2}-\lambda \int_{\Omega}|u|^{2} \ln |u| d x \\
& -\lambda \ln \lambda\|u\|^{2}-\frac{\lambda}{2}\|u\|^{2}+\frac{\lambda}{2}\|u\|^{2} \\
= & \lambda\left(\left\|A^{\frac{1}{2}} u\right\|^{2}-\int_{\Omega}|u|^{2} \ln |u| d x-\ln \lambda\|u\|^{2}\right) .
\end{aligned}
$$

Moreover, by taking

$$
\lambda^{*}=\lambda^{*}(u)=\exp \left(\frac{\left\|A^{\frac{1}{2}} u\right\|^{2}-\int_{\Omega}|u|^{2} \ln |u| d x}{\|u\|^{2}}\right)
$$

By (2.2), we get

$$
\begin{aligned}
I(\lambda u) & =\left\|A^{\frac{1}{2}}(\lambda u)\right\|^{2}-\int_{\Omega}|\lambda u|^{2} \ln |\lambda u| d x \\
& =\lambda^{2}\left\|A^{\frac{1}{2}} u\right\|^{2}-\lambda^{2} \int_{\Omega}|u|^{2} \ln |u| d x-\lambda^{2} \ln \lambda\|u\|^{2} \\
& =\lambda j^{\prime}(\lambda) .
\end{aligned}
$$

So, $I(\lambda u)>0$ for $\lambda \in\left(0, \lambda^{*}\right), I(\lambda u)<0$ for $\lambda \in\left(\lambda^{*},+\infty\right)$ and $I\left(\lambda^{*} u\right)=0$. Therefore, the proof is completed.

Lemma 2.4. $d$ defined by (2.4) is positive and there exists a positive function $u \in \mathcal{N}$ such that $J(u)=d$.

Proof. Let $\left\{u_{r}\right\}_{r}^{\infty} \subset \mathcal{N}$ be a minimizing sequence for $J$, which means that

$$
\lim _{r \rightarrow \infty} J\left(u_{r}\right)=d .
$$

We can easy show that $\left\{\left|u_{r}\right|\right\}_{r} \subset \mathcal{N}$ is also a minimizing sequence for $J$ due to $\left|u_{r}\right| \in \mathcal{N}$ and $J\left(\left|u_{r}\right|\right)=J\left(u_{r}\right)$. Therefore, we can suppose that $u_{r}>0$ a.e. $\Omega$ for all $r \in N$.

Moreover, we have already observed that $J$ is coercive on $\mathcal{N}$ which satisfies that $\left\{u_{r}\right\}_{r}^{\infty}$ is bounded in $H_{0}^{m}(\Omega)$. Let $\mu>0$ be small enough such that $2+\mu<\frac{2 n}{n-2}$. Since $H_{0}^{m}(\Omega) \hookrightarrow L^{2+\mu}(\Omega)$ is compact, so there exists a function $u$ and a subsequence of $\left\{u_{r}\right\}_{r}^{\infty}$, still denote by $\left\{u_{r}\right\}_{r}^{\infty}$, such that

$$
\begin{gathered}
u_{r} \rightarrow u \text { weakly in } H_{0}^{m}(\Omega), \\
u_{r} \rightarrow u \text { strongly in } L^{2+\mu}(\Omega), \\
u_{r}(x) \rightarrow u(x) \text { a.e. in } \Omega .
\end{gathered}
$$


Also, $u \geq 0$ a.e. in $\Omega$. First, we prove $u \neq 0$. From the dominated convergence theorem, we have

$$
\int_{\Omega}|u|^{2} \ln |u| d x=\lim _{r \rightarrow \infty} \int_{\Omega}\left|u_{r}\right|^{2} \ln \left|u_{r}\right| d x,
$$

and

$$
\int_{\Omega}|u|^{2} d x=\lim _{r \rightarrow \infty} \int_{\Omega}\left|u_{r}\right|^{2} d x .
$$

From the weak lower semicontinuity of $H_{0}^{m}(\Omega)$, we get

$$
\left\|A^{\frac{1}{2}} u\right\|^{2} \leq \liminf _{r \rightarrow \infty}\left\|A^{\frac{1}{2}} u_{r}\right\|^{2} .
$$

Then it follows from $(2.1),(2.10),(2.11),(2.12)$ and (2.13) that

$$
\begin{aligned}
J(u) & =\frac{1}{2}\left\|A^{\frac{1}{2}} u\right\|^{2}-\frac{1}{2} \int_{\Omega}|u|^{2} \ln |u| d x+\frac{1}{4}\|u\|^{2} \\
& \leq \liminf _{r \rightarrow \infty} \frac{1}{2}\left\|A^{\frac{1}{2}} u_{r}\right\|^{2}-\lim _{r \rightarrow \infty} \frac{1}{2} \int_{\Omega}\left|u_{r}\right|^{2} \ln \left|u_{r}\right| d x+\lim _{r \rightarrow \infty} \frac{1}{4}\left\|u_{r}\right\|^{2} \\
& =\liminf _{r \rightarrow \infty}\left(\frac{1}{2}\left\|A^{\frac{1}{2}} u_{r}\right\|^{2}-\frac{1}{2} \int_{\Omega}\left|u_{r}\right|^{2} \ln \left|u_{r}\right| d x+\frac{1}{4}\left\|u_{r}\right\|^{2}\right) \\
& =\liminf _{r \rightarrow \infty} J\left(u_{r}\right)=d .
\end{aligned}
$$

Using (2.2), (2.11) and (2.13), we have

$$
\begin{aligned}
I(u) & =\left\|A^{\frac{1}{2}} u\right\|^{2}-\int_{\Omega}|u|^{2} \ln |u| d x \\
& \leq \liminf _{r \rightarrow \infty}\left\|A^{\frac{1}{2}} u_{r}\right\|^{2}-\lim _{r \rightarrow \infty} \int_{\Omega}\left|u_{r}\right|^{2} \ln \left|u_{r}\right| d x \\
& =\liminf _{r \rightarrow \infty}\left(\left\|A^{\frac{1}{2}} u_{r}\right\|^{2}-\int_{\Omega}\left|u_{r}\right|^{2} \ln \left|u_{r}\right| d x\right) \\
& =\liminf _{r \rightarrow \infty} I\left(u_{r}\right)=0 .
\end{aligned}
$$

Since $u_{r} \in \mathcal{N}$, we have $I\left(u_{r}\right)=0$. So, by Lemma 1 and the fact $x^{-\mu} \ln x \leq(e \mu)^{-1}$ for $x \geq 1$, we get

$$
\begin{aligned}
\left\|A^{\frac{1}{2}} u_{r}\right\|^{2} & =\int_{\Omega}\left|u_{r}\right|^{2} \ln \left|u_{r}\right| d x \\
& \leq(e \mu)^{-1} \int_{\Omega}\left|u_{r}\right|^{2+\mu} d x \\
& =(e \mu)^{-1}\left\|u_{r}\right\|_{2+\mu}^{2+\mu} \\
& \leq C\left\|A^{\frac{1}{2}} u_{r}\right\|_{2}^{2+\mu},
\end{aligned}
$$

where $C$ is Sobolev emdedding constant. This satisfies that

$$
\int_{\Omega}\left|u_{r}\right|^{2} \ln \left|u_{r}\right| d x=\left\|A^{\frac{1}{2}} u_{r}\right\|^{2} \geq C .
$$

By (2.11) and (2.16), we have

$$
\int_{\Omega}|u|^{2} \ln |u| d x \geq C .
$$


Thus, we have $u \in H_{0}^{m}(\Omega) \backslash\{0\}$.

If $I\left(u_{r}\right)<0$, from Lemma 3, there exists a $\lambda^{*}$ such that $I\left(\lambda^{*} u\right)=0$ and $0<$ $\lambda^{*}<1$. Thus, $\lambda^{*} u \in \mathcal{N}$. It follows from (2.3), (2.4), (2.12) and (2.13) that

$$
\begin{aligned}
d & \leq J\left(\lambda^{*} u\right) \\
& =\frac{1}{2} I\left(\lambda^{*} u\right)+\frac{1}{4}\left\|\lambda^{*} u\right\|^{2} \\
& =\frac{\left(\lambda^{*}\right)^{2}}{4}\|u\|^{2} \\
& \leq\left(\lambda^{*}\right)^{2} \liminf _{r \rightarrow \infty} \frac{1}{4}\left\|u_{r}\right\|^{2} \\
& =\left(\lambda^{*}\right)^{2} \liminf _{r \rightarrow \infty} J\left(u_{r}\right) \\
& =\left(\lambda^{*}\right)^{2} d,
\end{aligned}
$$

which indicates $\lambda^{*} \geq 1$ by $d>0$. It contradicts $0<\lambda^{*}<1$. By (2.15), we have $I(u)=0$. For this reason, $u \in \mathcal{N}$. From (2.10), we have $J(u) \geq d$. From (2.14), we have $J(u) \leq d$. So, $J(u)=d$.

\section{MAIN RESUlts}

Definition 3.1. (Maximal Existence Time). Assume that $u(t)$ be weak solutions of problem (1.1). We define the maximal existence time $T_{\max }$ as follows

(i) If $u(t)$ exists for all $0 \leq t<\infty$, then $T_{\max }=+\infty$;

(ii) If there exists a $t_{0} \in(0, \infty)$ such that $u(t)$ exists for $0 \leq t<t_{0}$, but doesn't exists at $t=t_{0}$, then $T_{\max }=t_{0}$.

Definition 3.2. (Blow-up at $+\infty$ ). Let $u(t)$ be a weak solution of (1.1). We call $u(t)$ blow-up at $+\infty$ if the maximal existence time $T_{\max }=+\infty$ and

$$
\lim _{t \rightarrow+\infty}\|u(t)\|^{2}=+\infty .
$$

Theorem 3.3. Assume that $u_{0} \in H_{0}^{m}(\Omega) \backslash\{0\}, J\left(u_{0}\right)<d$ and $I\left(u_{0}\right)<0$. Let $u(t)$ be a weak solution to the problem (1.1). Then $u(t)$ blows up at $+\infty$ such that

$$
\lim _{t \rightarrow+\infty}\|u(t)\|^{2}=\infty
$$

Proof. Let $u(t)$ be weak solution of $(1.1)$ with $J\left(u_{0}\right)<d$ and $I\left(u_{0}\right)<0$. Let $F$ : $[0, \infty) \rightarrow \mathbb{R}^{+}$, and

$$
F(t)=\int_{0}^{t}\|u(s)\|^{2} d s
$$

Then, a direct calculation gives

$$
F^{\prime}(t)=\|u(t)\|^{2} .
$$

From (2.2) and (3.2), we get

$$
\begin{aligned}
F^{\prime \prime}(t) & =2 \int_{\Omega} u u_{t} d x \\
& =2 \int_{\Omega} u^{2} \ln |u| d x-2 \int_{\Omega} A u^{2} d x \\
& =-2 I(u)
\end{aligned}
$$


By (3.2) and (3.3), we get

$$
\begin{aligned}
F^{\prime}(t) \ln F^{\prime}(t)-F^{\prime \prime}(t) & =\|u(t)\|^{2} \ln \|u(t)\|^{2}+2 I(u) \\
& =2\|u(t)\|^{2} \ln \|u(t)\|+2\left\|A^{\frac{1}{2}} u\right\|^{2}-2 \int_{\Omega}|u|^{2} \ln |u| d x \\
& \geq 0,
\end{aligned}
$$

which, in turn, yields that

$$
\left(\ln F^{\prime}(t)\right)^{\prime} \leq \ln F^{\prime}(t)
$$

This means

$$
\ln F^{\prime}(t) \leq e^{t} \ln F^{\prime}(0)=e^{t} \ln \left\|u_{0}\right\|^{2} .
$$

Then

$$
\|u(t)\|^{2} \leq\left\|u_{0}\right\|^{e^{t}}, \quad \forall t \geq 0,
$$

which yields that $u(t)$ does not blow up in finite time.

On the other hand, using the Hölder inequality and combining (3.3), we have

$$
\begin{aligned}
\frac{1}{4}\left(F^{\prime}(t)\right)^{2} & =\frac{1}{4}\left(\int_{0}^{t} F^{\prime \prime}(s) d s\right)^{2} \\
& =\left(\int_{0}^{t} \int_{\Omega} u u_{s} d x d s\right)^{2} \\
& \leq \int_{0}^{t}\|u(s)\|^{2} d s \int_{0}^{t}\left\|u_{s}\right\|^{2} d s .
\end{aligned}
$$

From (2.3) and (3.3), it follows

$$
\begin{aligned}
F^{\prime \prime}(t) & =-2 I(u) \\
& =-4 J(u)+\|u\|^{2} \\
& \geq-4 J\left(u_{0}\right)+4 \int_{0}^{t}\left\|u_{s}(s)\right\|^{2} d s+\|u\|^{2} .
\end{aligned}
$$

By Lemma 3, there exists a $\lambda^{*} \in(0,1)$ such that $I\left(\lambda^{*} u(t)\right)=0$. Thus, by the definition of $d$, it follows that

$$
\begin{aligned}
d & =\inf _{u \in \mathcal{N}} J(u) \leq J\left(\lambda^{*} u(t)\right) \\
& =\frac{1}{2} I\left(\lambda^{*} u(t)\right)+\frac{1}{4}\left\|\lambda^{*} u(t)\right\|^{2} \\
& =\frac{1}{2} I\left(\lambda^{*} u(t)\right)+\frac{\left(\lambda^{*}\right)^{2}}{4}\|u(t)\|^{2} \\
& \leq \frac{1}{4}\|u(t)\|^{2} .
\end{aligned}
$$

Combining (3.5) and (3.6), we have

$$
\begin{aligned}
F^{\prime \prime}(t) & \geq-4 J\left(u_{0}\right)+4 \int_{0}^{t}\left\|u_{s}(s)\right\|^{2} d s+\|u\|^{2} \\
& \geq 4\left(d-J\left(u_{0}\right)\right)+4 \int_{0}^{t}\left\|u_{s}(s)\right\|^{2} d s .
\end{aligned}
$$


Using (3.1), (3.4) and (3.7), we get

$$
\begin{aligned}
F(t) F^{\prime \prime}(t) & \geq 4\left(d-J\left(u_{0}\right)\right) F(t)+4 \int_{0}^{t}\|u(s)\|^{2}\left\|u_{s}(s)\right\|^{2} d s \\
& \geq 4\left(d-J\left(u_{0}\right)\right) F(t)+\left(F^{\prime}(t)\right)^{2} .
\end{aligned}
$$

Then, we see that

$$
F(t) F^{\prime \prime}(t)-\left(F^{\prime}(t)\right)^{2} \geq 4\left(d-J\left(u_{0}\right)\right) F(t) .
$$

By $J\left(u_{0}\right)<d$ and $I(u)<0$, then we know

$$
F(t) F^{\prime \prime}(t)-\left(F^{\prime}(t)\right)^{2}>0 .
$$

On the other hand, by straightforward calculation, it is clear that

$$
(\ln F(t))^{\prime}=\frac{F^{\prime}(t)}{F(t)}
$$

and

$$
(\ln F(t))^{\prime \prime}=\frac{F(t) F^{\prime \prime}(t)-\left(F^{\prime}(t)\right)^{2}}{(F(t))^{2}}>0 .
$$

From (3.10), we know that $(\ln F(t))^{\prime}$ is increasing with respect to $t$, using this fact, integrating (3.9) from $t_{0}$ to $t$, we get

$$
(\ln F(t))^{\prime}=\left(\ln F\left(t_{0}\right)\right)^{\prime}+\int_{t_{0}}^{t} \frac{F(s) F^{\prime \prime}(s)-\left(F^{\prime}(s)\right)^{2}}{(F(s))^{2}} d s,
$$

and

$$
\begin{aligned}
\ln F(t)-\ln F\left(t_{0}\right) & =\int_{t_{0}}^{t}(\ln F(s))^{\prime} d s \\
& =\int_{t_{0}}^{t} \frac{F^{\prime}(s)}{F(s)} d s \\
& \geq \frac{F^{\prime}\left(t_{0}\right)}{F\left(t_{0}\right)}\left(t-t_{0}\right),
\end{aligned}
$$

where $0 \leq t_{0} \leq t$. Then

$$
F(t) \geq F\left(t_{0}\right) \exp \left(\frac{F^{\prime}\left(t_{0}\right)}{F\left(t_{0}\right)}\left(t-t_{0}\right)\right) .
$$

Since $F(0)=0$ and $F^{\prime}(0)>0$, we can take $t_{0}$ small enough such that $F^{\prime}\left(t_{0}\right)>0$ and $F\left(t_{0}\right)>0$. Then for sufficiently large $t$,

$$
\begin{aligned}
\|u(t)\|^{2} & =F^{\prime}(t) \\
& \geq \frac{F^{\prime}\left(t_{0}\right)}{F\left(t_{0}\right)} F(t) \\
& \geq F^{\prime}\left(t_{0}\right) \exp \left(\frac{F^{\prime}\left(t_{0}\right)}{F\left(t_{0}\right)}\left(t-t_{0}\right)\right) \\
& =\left\|u\left(t_{0}\right)\right\|^{2} \exp \left(\frac{F^{\prime}\left(t_{0}\right)}{F\left(t_{0}\right)}\left(t-t_{0}\right)\right) \\
& \geq\left\|u_{0}\right\|^{2} \exp \left(\frac{F^{\prime}\left(t_{0}\right)}{F\left(t_{0}\right)}\left(t-t_{0}\right)\right), \quad t \geq t_{0},
\end{aligned}
$$


i.e.,

$$
\lim _{t \rightarrow+\infty}\|u(t)\|^{2}=+\infty .
$$

This shows that weak solution $u(t)$ of the problem (1.1) blows up at $+\infty$.

\section{Conclusion}

In this paper, we examined the initial boundary value problem for a higher-order parabolic equation with logarithmic nonlinearity. We obtained blow-up at infinity of weak solution, by using the potential well method and logarithmic convexity method.

\section{Acknowledgments}

The authors would like to thank the reviewers and editors of Journal of Universal Mathematics.

\section{Funding}

The author(s) declared that has no received any financial support for the research, authorship or publication of this study.

The Declaration of Conflict of Interest/ Common Interest

The author(s) declared that no conflict of interest or common interest

\section{The Declaration of Ethics Committee Approval}

This study does not be necessary ethical committee permission or any special permission.

\section{The Declaration of Research and Publication Ethics}

The author(s) declared that they comply with the scientific, ethical, and citation rules of Journal of Universal Mathematics in all processes of the study and that they do not make any falsification on the data collected. Besides, the author(s) declared that Journal of Universal Mathematics and its editorial board have no responsibility for any ethical violations that may be encountered and this study has not been evaluated in any academic publication environment other than Journal of Universal Mathematics.

\section{REFERENCES}

[1] R.A. Adams and J.J.F. Fournier, Sobolev Spaces, Academic Press, (2003).

[2] H. Chen, P. Luo, G. Liu, Global solution and blow-up of a semilinear heat equation with logarithmic nonlinearity, Journal of Mathematical Analysis and Applications, 422(1), 84-98, (2015).

[3] H. Chen, S. Tian, Initial boundary value problem for a class of semilinear pseudo-parabolic equations with logarithmic nonlinearity, Journal of Differential Equations, 258, 4424-4442, (2015).

[4] V.A. Galaktionov, Critical global asymptotics in higher-order semilinear parabolic equations, International Journal of Mathematics and Mathematical Sciences, 60, 3809-3825, (2003).

[5] Y. Han, Blow-up at infinity of solutions to a semilinear heat equation with logarithmic nonlinearity, Journal of Mathematical Analysis and Applications, 471, 513-517, (2019).

[6] Y. He, H. Gao, H. Wang, Blow-up and decay for a class of pseudo-parabolic p-Laplacian equation with logarithmic nonlinearity, Computers \& Mathematics with Applications, 75, 459469, (2018). 
[7] K. Ishige, T. Kawakami, S. Okabe, Existence of solutions for a higher-order semilinear parabolic equation with singular initial data, Annales de l'Institut Henri Poincare C, Analyse Nonlineaire, 37, 1185-1209, (2020).

[8] P. Li, C. Liu, A class of fourth-order parabolic equation with logarithmic nonlinearity, Journal of Inequalities and Applications, 328, 1-21, (2018).

[9] L.C. Nhan, L.X. Truong, Global solution and blow-up for a class of pseudo p-Laplacian evolution equations with logarithmic nonlinearity, Computers \& Mathematics with Applications, 73, 2076-2091, (2017).

[10] J. Peng, J. Zhou, Global existence and blow-up of solutions to a semilinear heat equation with logarithmic nonlinearity, Applicable Analysis, 1-21, (2019).

[11] E. Pişkin, N. Polat, On the decay of solutions for a nonlinear higher-order Kirchhoff-type hyperbolic equation, Journal of Advanced Research in Applied Mathematics, 5(2), 107-116, (2013).

[12] E. Pişkin, Blow up solutions for a class of nonlinear higher-order wave equation with variable exponents, Sigma Journal of Engineering and Natural Sciences, 10(2), 149-156, (2019).

[13] L. Xiao, M. Li, Initial boundary value problem for a class of higher-order $n$-dimensional nonlinear pseudo-parabolic equations, Boundary Value Problems, 5, 1-24, (2021).

[14] Y. Ye, Existence and asymptotic behaviour of global solutions for a class of nonlinear higherorder wave equation, Journal of Inequalities and Applications, 1-14, (2010).

[15] J. Zhou, X. Wang, X. Song, C. Mu, Global existence and blowup of solutions for a class of nonlinear higher-order wave equations, Zeitschrift für Angewandte Mathematik und Physik, 63, 461-473, (2012).

(Tuğrul Cömert (Corresponding author)) Dicle University, Department of Mathematics, DIYARBAKIR, TURKEY

Email address: tugrulcomertt@gmail.com

(Erhan Pişkin) Dicle University, Department of Mathematics, 21280, Diyarbakir, Turkey

Email address: episkin@dicle.edu.tr 